\title{
BMJ Open VOICES: the value of 6-month clinical evaluation in stroke. The protocol for a planned qualitative study to ascertain the value of stroke follow-up to people affected by stroke
}

\author{
Colin Jenkins, ${ }^{1}$ Fiona Price ${ }^{2}$
}

To cite: Jenkins C, Price $F$. VOICES: the value of 6 month clinical evaluation in stroke. The protocol for a planned qualitative study to ascertain the value of stroke follow-up to people affected by stroke. BMJ Open 2014;4: e006384. doi:10.1136/ bmjopen-2014-006384

- Prepublication history for this paper is available online. To view these files please visit the journal online (http://dx.doi.org/10.1136/ bmjopen-2014-006384).

Received 14 August 2014 Revised 1 October 2014 Accepted 3 October 2014

CrossMark

\footnotetext{
${ }^{1}$ Geriatric Medicine and Stroke Services Herefordshire NHS, Wye Valley NHS Trust, The County Hospital, Hereford, Herefordshire, UK ${ }^{2}$ Stroke Team Office, Wye Valley NHS Trust, The County Hospital, Hereford, Herefordshire, UK
}

Correspondence to Dr Fiona Price; fiona.price@wvt.nhs.uk

\section{ABSTRACT}

Introduction: The National Clinical Guidelines for Stroke recommend 'routine follow-up of patients 6 months post discharge'. The Sentinel Stroke National Audit Programme sets a standard of 6 months postadmission follow-up, capturing data on process and outcomes. There appears to be no convincing model of stroke follow-up at 6 months, and despite evidence of unmet need in almost $50 \%$ of stroke survivors $1-5$ years after their stroke, little work focuses on the first 12 months of recovery. By listening to the living experiences of stroke, the research aims to tailor the stroke care pathway to the needs of those affected. Methods and analysis: A focus group of six stroke survivors and carers will be invited to identify appropriate interview questions about the value of follow-up at 6 months, ensuring that this study has its genesis in the participant experience. A pilot study of four stroke survivors will ascertain the feasibility of the method. Thirty stroke survivors from the follow-up clinic will be invited to take part in semistructured interviews. Raw data, in the form of digital recordings of the interviews, will be transcribed. Interview transcriptions will be checked by the participant for accuracy prior to analysis using NVivo software. Literal and reflective narrative analysis will be used to code transcribed text to examine shared themes and reflect on content.

Ethics and dissemination: Study documentation has been reviewed by the Coventry and Warwickshire Research Ethics Committee; the chief investigator met with the committee to scrutinise the study and justify its methodology. The committee has approved this study. A copy of the final report will be given to participants, the Stroke Association, the local Clinical Commissioning Group and participants' general practitioners. It is intended to disseminate the results locally by presentation to the Trust board, at academic conferences and by publication in a peer-reviewed scientific journal.

\section{BACKGROUND AND RATIONALE}

The National Clinical Guidelines for Stroke recommend 'routine follow-up of patients

\section{Strengths and limitations of this study}

- Patient and carer involvement-interview questions arise from stroke survivors, not from the researcher's assumption.

- Detailed, holistic data.

- Lived and living, active histories.

- Geographically specific, but may be generalisable to others in similar locations.

- Relatively small sample size, but close to $30 \mathrm{~h}$ of data is broad and deep, and should providing vivid, compelling accounts.

- Subjectivity of qualitative style is inevitable and acknowledged.

6 months post discharge and annually after a stroke' and 'any patient with residual impairment after the end of initial rehabilitation should be offered a formal review at least every 6 months, to consider whether further interventions are warranted'. These recommendations are a consensus view of the expert working party. ${ }^{1}$

The Sentinel Stroke National Audit Programme (SSNAP) sets a standard of 6 months postadmission follow-up assessment ( \pm 2 months): this captures data on process and some outcomes. ${ }^{2}$

There appears to be no convincing theoretical model of stroke follow-up at 6 months; there is also a notable discrepancy between 6 months postadmission and 6 months postdischarge recommended by SSNAP and the National Clinical Guidelines for Stroke respectively. Two studies suggest that some patients will benefit from physiotherapy. ${ }^{3} 4$ Forster et al however found no evidence for a structured reassessment at 6 months in terms of resource usage.

Some person-centred qualitative evidence is available from the Stroke Association 
survey 'Feeling Overwhelmed: the emotional impact of stroke' of 2700 people affected by stroke. ${ }^{6}$ This research examined the emotional impact of stroke on survivors, their carers and families and highlighted the need for further research into what they describe as an 'underappreciated problem'. ${ }^{6}$ Finally, Martin Gower highlighted the need to focus on service user and carer involvement in helping to shape the stroke care agenda in the Comprehensive Local Research Network (CLRN) 'Celebrating Achievements' conference. ${ }^{7}$

\section{PATIENT BENEFIT}

There is evidence of unmet need in almost $50 \%$ of stroke survivors between 1 and 5 years after the stroke ${ }^{8}$ though little work focuses on the first 12 months of recovery. Our 6 month consultant-led follow-up clinic currently examines the needs of local patients and their carers.

This study aims to provide patient benefit by having a positive impact on the short-term to medium-term holistic physical and psychological well-being of the patient and their carers. By ascertaining the value of follow-up intervention from the stroke survivor's perspective, we aim to provide a beneficial service tailored to the needs of individuals. The provision of a follow-up service at 6 months has been timed according to the National Clinical Guidelines for Stroke $^{1}$ but there is no clear evidence to show why 6 months has been chosen as an appropriate time. This study will ascertain the value of the follow-up directly from the living experiences of the stroke survivors and carers as experts, and could lead to evidence for follow-up at a different time. Results from this study could be incorporated into the National Clinical Guidelines for Stroke and ultimately achieve benefit for all users of stroke services within the National Health Service (NHS).

\section{OBJECTIVES}

\section{Principle objectives}

- What is the value to people affected by stroke of a 6 month follow-up clinic?

- Is 6 months poststroke the best time?

Secondary objectives

- The study will systematically review previous research in this area and seek to fill the specific gap in knowledge about the value of follow-up.

- The study will follow a given methodology, a patientcentred, constructivist qualitative philosophy, in order to collect robust data.

- The term 'value' will be examined to determine how it is perceived and interpreted by stroke survivors.

- Data will be examined using narrative analysis to gain the lived and living experience of stroke survivors.

- Results will inform local stroke provision.

\section{TRIAL DESIGN, METHODOLOGY AND METHOD}

This is a qualitative study using a convenience sample. The philosophy is to use a qualitative, constructivist, interpretive method to co-construct knowledge about the value of follow-up, since a person-centred approach was not the focus of previous studies assessing the value of stroke follow-up. It is intended that this study will provide valuable evidence to inform decisions about local stroke service development. This is particularly important as there has been limited service user engagement in service design previously.

The study will use a convenience sampling method, since people affected by stroke will be approached in the clinic offered at the hospital. There will be no selection by the researchers; all those who attend will be offered the chance to take part regardless of age, ability or any other criteria other than those exclusion criteria listed. Carers or relatives of stroke survivors who could act as interpreters would be welcome to participate in the study with the individual.

Potential participants will be approached at the end of their 6 month clinical follow-up appointment and invited to participate within the next 2 weeks. Written information about the study and a contact number will be given out if interest is initially expressed. Within the next week a researcher will make contact to invite formal enrolment, gain consent and arrange the interview date, time and venue. It is intended to hold interviews within 2 weeks of the clinic appointment. This will allow participants to prepare for the interview by making notes or reflecting on what they valued in the follow-up. The aim is to recruit up to 30 stroke survivors into the study.

A semistructured interview will be conducted in the hospital or person's home, depending on their choice. Interviews will last a maximum of $1 \mathrm{~h}$. As the interview style is semistructured, the length of interview and depth of information proffered will be determined by the participant. This style of interview allows the participant to offer as much or little detail as they see fit, since the topic is likely to require some emotional investment from each individual. The emotional state and vulnerability of the individual will be considered, so interviews could be staged into short time sections in order not to tire the individual and to encourage the participant to feel they were needed and not 'being used'. The physical and psychological safety of the participant will be paramount. If a participant should disclose information which was of concern to the interviewer, the interviewer will follow the multiagency safeguarding adults policy agreed by the local Adult Safeguarding Board.

The interviews will be digitally recorded and transcribed, then stored on-line in a password-protected file only accessible by the researchers and one secretary. Transcriptions will be analysed using NVivo software. The use of qualitative software will standardise analysis, resulting in broad themes which can be interpreted and illustrated using verbatim quotations.

Narrative analysis will be used to code transcribed text to examine themes and key words from the raw data. Narrative analysis is an examination of individual stories that can contribute to an understanding of that 
individual's experience. In this case the 'stories' are the content of the interview, the lived and living experience of stroke as described by the stroke survivor in the context of an interview conversation. Narrative analysis, in which experiences are constructed from dialogic aspects of narrative ${ }^{9}$ can examine data from a literal or reflexive approach. Both will be used in this study; literal analysis will examine particular language, for example repeated words or phrases, and reflexive analysis will include the researchers' and participants' contribution to the cocreation of knowledge through the interpretation and reflection on content. ${ }^{10}$ Verbatim quotes will be used to illustrate themes or recurrent points.

All data will be anonymised and potential participants will be advised of this when giving informed consent. Participants will also be offered the opportunity to validate the transcription by checking a copy of the transcribed interview for accuracy. Transcriptions will be posted or emailed to the participant, whichever method they prefer, and the researcher practitioner's contact number and email will be provided for them to call or email with their comments. They will be advised that they are being asked to ensure that the transcription is an accurate record of their interview and to confirm again that they are happy for quotes to be used in the final report. This process of validation will give participants ownership of the data and further allow them to agree to its use. This collaborative approach will enable the co-construction of new knowledge between the researchers, and the participants as experts.

The full study is expected to last 2 years, with a focus group and pilot interviews taking place in the first year. Transcription, data analysis and report writing are anticipated to be completed in the second year.

\section{ELIGIBILITY}

Inclusion

- Those attending the 6 month follow-up clinic appointment.

- Adults over the age of 18 .

- Able to give informed consent, or proxy consent from a relative.

- Individuals with aphasia may take part if they have a close relative who can help make their views understood through verbal or written means.

\section{Exclusion}

- Those who had a stroke less than 6 months ago.

- Age less than 18.

- Those who do not speak English fluently and who do not have an interpreter who can translate for them.

- Non-stroke life expectancy of less than 6 months.

- Individuals with dementia whose memory is impaired to a degree that they could not give meaningful consent.

- Individuals who do not have capacity to consent.

\section{RECRUITMENT}

The trial uses an opportunistic sampling strategy. Potential participants will be approached at the end of their clinical follow-up by the chief investigator (who runs the clinic) and invited to participate within the next 2 weeks. Written information about the study and the contact number of the researcher will be given out if interest is initially expressed. Written information using text that has key words and concepts in bold ${ }^{11}$ will be provided to those with dysphasia to enable them to express their own wishes about participation. Within the next week the researcher will make contact to invite formal enrolment, gain consent and arrange the interview date, time and venue. The researcher will not be present in the clinic interview.

\section{CONSENT}

Informed, written consent will be sought for all participants. When initial interest is expressed, individuals will be given an information sheet and contact telephone numbers to take away with them. The researcher will gain written consent before the interview takes place. The participant will be given a copy of their signed consent form. Hard copies of consent forms will be stored securely at the study centre.

\section{DATA SOURCES AND MEASUREMENT}

Raw data will be in the form of digital recordings of the interviews. These will be transcribed to enable analysis to be completed efficiently. Transcribed interviews will be identified by a numerical code unique to each individual. Transcriptions will be analysed using NVivo software. In response to ethics committee recommendations, transcriptions will only be entered into the NVivo analysis software by FP, (ie, before any analysis or coding takes place) since there is potential to bias the results if the researcher who runs the clinic (CJ) also sees the interview content. The transcriptions will have been checked by the participant for accuracy prior to analysis.

Coded 'chunks' of data will be analysed by both researchers and there will be an iterative process of reflection on content by both researchers. Literal and reflective narrative analysis will be used to code transcribed text to examine shared themes and key words, and reflect on the content of the interviews. Verbatim quotes will be used to illustrate themes or recurrent points.

\section{QUALITY ASSURANCE}

The chief investigator and coinvestigator have valid Good Clinical Practice certificates and are experienced researchers. The scientific quality of the study has been assessed by independent peer-review of the proposal by a university lecturer, via the West Midlands South Comprehensive Local Research Network Research and Development team. It has also been scrutinised by the Trust, acting as sponsor. In addition, this proposal has been reviewed by the Research and Development team and the Research Design Service at study preparation and prior to the start. 
Finally the proposal has also been considered by a member of the stroke team who is not involved in the research but who has extensive knowledge of stroke and experience of working with patients in a person-centred way.

\section{CONFIDENTIALITY}

Digital interview recordings, written transcriptions and written analysis will be kept in a file on a password protected secure NHS network drive. Access to this file will be restricted to both named researchers and one member of secretarial staff. All data will be anonymised and potential participants will be advised of this as part of the consent process. Hard copies of consent forms will be kept in a locked filing cabinet in a locked office.

\section{ACCRUAL AND ANALYSIS}

\section{Sample size up to 30}

The minimum recruitment is set at 12 to enable recruitment of one person a month, though it is expected that this will be exceeded. The maximum is calculated on the basis that saturation point will be reached, whereby no further new information will be gained by interviewing more participants. The data provided in interview will be rich and deep, so a relatively small sample size is justified.

\section{ANALYSIS METHODS}

A narrative style of analysis will be used to examine shared themes and commonality in the interview transcriptions. NVivo software will be used to standardise the analysis. Narrative analysis centres on the structured study of stories or oral narrative accounts of complex and nuanced experience, in this case taking the form of interview responses. Individual interview stories can be categorised and analysed by themes within the account (thematic analysis) or by the way the interview conversation is structured; for example examining the use of metaphor would result in a structural analysis of the narrative. It is anticipated that both types of analysis will be used in this study.

There is the potential for the chief investigator to be biased against any negative narratives arising from interviews since it is his clinic under scrutiny. In order to mitigate this possibility, raw data will be entered into the NVivo qualitative software package by FP, removing the need for the chief investigator to examine raw data. Analysis of coded, processed data will then be undertaken by both researchers in order to answer the research question.

\section{LONG-TERM STORAGE OF DATA}

Digital voice recordings of interviews, transcribed interviews and analysed data will be kept for 5 years after publication, and then destroyed. The rationale for keeping data for this length of time is to allow sufficient time for publication of the research in a peer-reviewed journal and subsequent academic review.

\section{PATIENT AND PUBLIC INVOLVEMENT}

The National Clinical Guidelines for Stroke ${ }^{1}$ advise that the views of patients with stroke and their carers should be considered when evaluating a service, and this study aims to answer that call.

A focus group of six stroke survivors and their carers will be invited to discuss the study and proposed method to ensure that the approach is appropriate and robust. The focus group discussion will also be used to devise and validate the interview questions, to ensure that attention is paid to the views and feelings of stroke survivors. Members of the focus group will consist of individuals who will not have attended a recent follow-up appointment so they will be able to approach the study from an independent viewpoint. Group members will be asked for their permission to have the discussion recorded. The content and feedback from this discussion will enable the focus of the resulting research questions to be precisely based on the views of patients and carers, thereby ensuring that this study has its genesis in the participant experience, not the researcher's interpretation of what that experience may be.

Four stroke survivors who did not take part in the focus group will be recruited from the follow-up clinic to take part in a one-to-one pilot interview to ascertain the feasibility of the study method. Again, feedback will be sought from the pilot interviewees on the questions and the way the study was run, and final amendments to the full study will be made accordingly.

Participants in the pilot and main study will be offered the opportunity to validate the transcription by checking a copy of their interview for accuracy. This will give participants ownership of the data and further allow them to agree to its use. Participants will also be given a copy of the final report, to see the results of their involvement.

\section{ETHICAL CONSIDERATIONS}

The study proposal has been reviewed by the West Midlands South Comprehensive Local Research Network, and been peer-reviewed by an independent university lecturer who acted as a reviewer.

All study documentation has been reviewed by the Coventry and Warwickshire Research Ethics Committee and the chief investigator met with the committee to scrutinise the study and justify its methodology. The committee has approved this study.

Risk of breach of confidentiality will be minimised by the use of anonymised data. Participants will be asked to consent to direct quotations from interview being used in the final report, in the knowledge that they will not be named or their identity be inferred.

There is a minimal risk that people might become upset while talking about their experiences of stroke; FP is an experienced interviewer and will support people appropriately using active listening skills. People will not have to answer any questions they find uncomfortable and can withdraw at any time in the study; this will be made clear in the consent process. 
Figure 1 Prestudy timetable.

Pre-Study Timetable

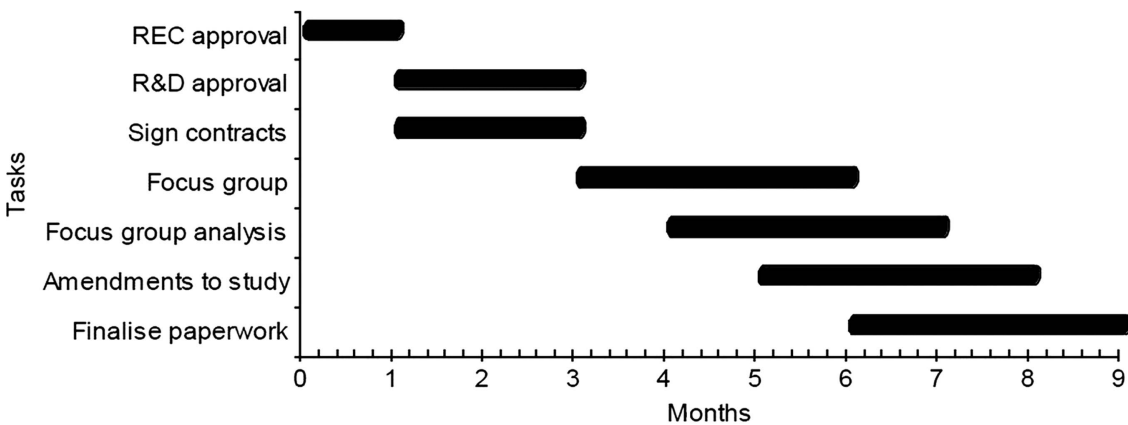

In the event of an individual becoming distressed, they will be asked if they wish to delay or discontinue the interview, and FP will ensure that someone is with the participant once the interview is completed. The interviewer will also be equipped to provide the participant with details of support organisations or help-lines should the need arise.

\section{ANTICIPATED PROBLEMS}

There might be a bias caused by the participant receiving additional attention by taking part in the interview; they might over-value the clinic appointment because additional attention has been paid to them and they place value on that process. Participants will be reminded that the focus of the study is the value of the clinic appointment, so as not to confound the results.

Individuals may be reluctant to offer negative views about the clinic if they are aware that those views will be fed back to the consultant. It will be made clear in the informed consent process that (1) data will not be attributable to them by name, (2) the consultant will not analyse raw data but will only examine the resulting themes, and (3) FP is not employed by the Trust and so she can offer an objective analysis of the results.

\section{POTENTIAL BENEFIT TO RESEARCH PARTICIPANTS}

For some individuals, participation may allow further opportunity to reflect on their development since their stroke or to take part in a worthwhile endeavour which could benefit others. In other words, participation may be beneficial since it enables them to have some influence or a role.

\section{EXPECTED OUTCOMES OF THE STUDY}

The study will inform the development of local stroke services in an area that has hitherto had little resource or clinical attention. The study will inform commissioners of the benefits to people affected by stroke of follow-up by stroke clinicians. The study will also enhance the theoretical basis for stroke follow-up. The study might show that there is no benefit to 6 month follow-up in its current SSNAP-based format but may suggest alternative approaches or timing of follow-up.

\section{DISSEMINATION OF RESULTS AND PUBLICATION POLICY}

A copy of the final study report will be given to the participants, participants' general practitioners, the Stroke Association and the local Clinical Commissioning Group. It is further intended to disseminate the results by presentation at academic conferences and by publication in a peer-reviewed scientific journal.

\section{TIMETABLE}

The timetable is subdivided into a prestudy set-up period, and the pilot and main study. Figure 1 shows the

Figure 2 Study timetable.

Study Timetable

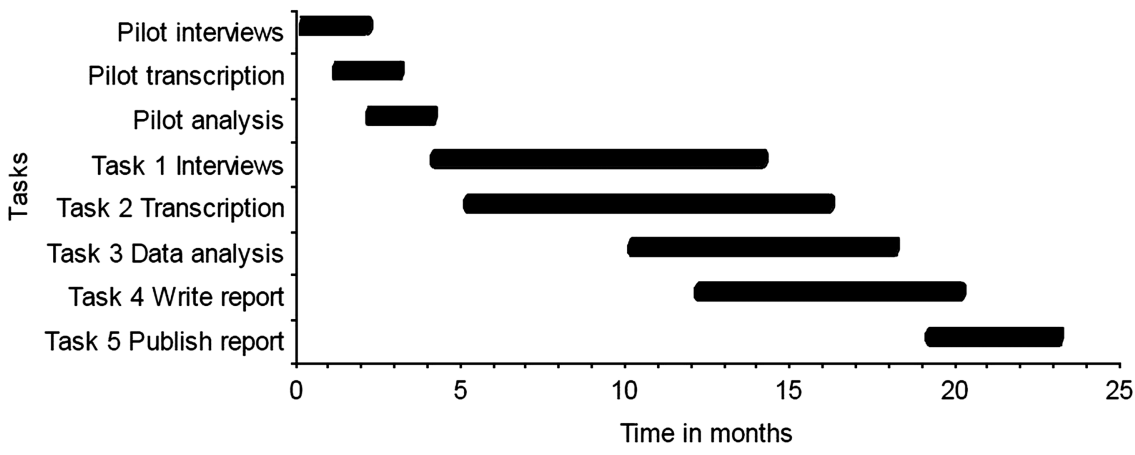




\begin{tabular}{llll}
\hline & Year 1 & Year 2 & Total \\
\hline Salaries (typing costs at £1.50 per minute) & $£ 1350^{*}$ & $£ 1350^{*}$ & $£ 1700$ \\
Equipment (external computer hard drive and digital encrypted dictaphone) $_{\text {Consumables (NVivo software) }}$ & $£ 390$ & - & $£ 390$ \\
Travel (researcher to patient homes, and participants to venue plus parking costs) & $£ 835$ & - & $£ 835$ \\
$\begin{array}{l}\text { Other expenses (printing information and consent forms; postage; literature: } \\
\text { printing and access costs) }\end{array}$ & $£ 400$ & - & $£ 400$ \\
Total & & & $£ 400$ \\
\hline
\end{tabular}

*The salary budget is designed to be used to pay one member of secretarial staff to transcribe interviews, and the typing cost is set to reflect the fact that transcription is in addition to their usual duties.

anticipated timing to gain approvals, run the focus group and amend the study based on focus group feedback.

Figure 2 shows the estimated timetable for the study, starting May 2014 and anticipated to end in May 2016. Both investigators expect to be in post for the duration of the study.

\section{BUDGET SUMIMARY}

Table 1 below shows the breakdown of the budget for the two years of the study. The budget is largely for initial capital costs to enable the study to be set up.

\section{TEAM EXPERTISE}

The CI has experience of acting as principal investigator for seven clinical stroke trials and has undertaken independent qualitative research in the past. The coinvestigator has successfully completed independent doctoral level qualitative study. Her post is funded by the NIHR Clinical Research Network. Both researchers hold current Good Clinical Practice certificates. In addition, both researchers have a person-centred focus and are motivated to gain the personal histories of people affected by stroke in order to inform service provision.

Acknowledgements The authors are very grateful to The Eveson Charitable Trust and The James Tudor Foundation for funding this study. Thanks also to Dr Karima Kadi-Hanifi and the anonymous reviewers for their suggestions. Finally, the authors acknowledge the contribution of Ciara Harris-without her word-play skills this study would have no name!

Contributors CJ originally conceived the idea for the study and has made a substantial contribution to the design and methodology of the research protocol. He has reviewed the protocol content, researched the background to the issue and collaborated in the writing of the full document. FP has drafted and revised the written protocol based on scrutiny by independent academic colleagues and has devised a structured method for the research.
Funding The study has been funded by two local charities, The Eveson Charitable Trust and The James Tudor Foundation.

\section{Competing interests None.}

Ethics approval Coventry and Warwickshire Research Ethics Committee.

Provenance and peer review Not commissioned; externally peer reviewed.

Open Access This is an Open Access article distributed in accordance with the Creative Commons Attribution Non Commercial (CC BY-NC 4.0) license, which permits others to distribute, remix, adapt, build upon this work noncommercially, and license their derivative works on different terms, provided the original work is properly cited and the use is non-commercial. See: http:// creativecommons.org/licenses/by-nc/4.0/

\section{REFERENCES}

1. Department of Health. The national clinical guidelines for stroke. London: HMSO, 2012.

2. Royal College of Physicians. Sentinel Stroke National Audit, Programme. 2013. https://www.strokeaudit.org/ (accessed 19 Jun 2013).

3. Duncan PW, Sullivan KJ, Behrman AL, et al. Body-weightsupported treadmill rehabilitation after stroke. $N$ Engl $J$ Med 2011;364:2026-36.

4. Ferrarello F, Baccini M, Rinaldi LA, et al. Efficacy of physiotherapy interventions late after stroke: a meta-analysis. J Neurol Neurosurg Psychiatry 2011;82:136-43.

5. Forster A, Young J, Green J, et al. Structured re-assessment system at 6 months after a disabling stroke: a randomised controlled trial with resource use and cost study. Age Ageing 2009;38:576-83.

6. Stroke Association. Feeling Overwhelmed: the emotional impact of stroke. 2013. http://www.stroke.org.uk/involved/ feeling-overwhelmed-report (accessed 19 Jun 2013).

7. Comprehensive Local Research Network conference. Celebrating, Achievements and Planning for the Future. 1 July 2013, Stratford, Manor Hotel, Stratford-upon-Avon.

8. McKevitt C, Fudge N, Refern J, et al. UK stroke survivors needs survey. London: Stroke, Association and Kings College London, 2011

9. Riessman CK. Narrative methods for the human sciences. Los Angeles, CA: Sage, 2008.

10. Welsh E. Dealing with data: using NVivo in the qualitative data analysis process. Forum Quant Soc Res 2002;3:Article 26.

11. Dalemans R, Wade DT, van den Heuvel WJA, et al. Facilitating the participation of people with aphasia in research: a description of strategies. Clin Rehabil 2009;23:948-59. 